\title{
HIF-1-modified BMSCs improve migration and reduce neuronal apoptosis after stroke in rats
}

\author{
YE ZhiZhong $^{1 \dagger}$, YE WeiBiao ${ }^{2 \dagger}$, DENG YuBin $^{2 *}$, WANG Jue ${ }^{2}$, ZHOU GuangQian $^{3} \&$ \\ ZHANG XiuQuan ${ }^{4}$ \\ ${ }^{1}$ Department of Rheumatology, The Futian Affiliated Hospital, Guangdong Medical College, Dongguan 518000, China; \\ ${ }^{2}$ Translational Medicine Laboratory, the First Affiliated Hospital, Sun Yat-sen University, Guangzhou 510080, China; \\ ${ }^{3}$ School of Medicine, Shenzhen University, Shenzhen 518061, China; \\ ${ }^{4}$ University of Utah School of Medicine, Salt Lake City, Utah 84132, USA
}

Received November 16, 2012; accepted February 5, 2013

Bone marrow-derived mesenchymal stem cells (BMSCs) have been demonstrated ameliorating neurologic deficits after stroke. Hypoxia-inducible factor-1 (HIF-1), the key regulator of cellular responses to low oxygen concentration, can activate multiple genes involving in crucial aspects for neurologic recovery. In this study, we present that rat BMSCs overexpression of $H I F-1 \alpha$ showed higher expression of HIF-1 target genes in HIF-1-BMSCs, including CXCR4, EPO, and VEGF. BMSCs-mHIF-1 $\alpha$ also exhibited an enhanced mobility towards the ischemic area within rat brain. Neural cell apoptosis in ischemic brain shown less severe in rats transplanted with HIF-1-BMSCs. Furthermore, the number of cells expressing neural progenitor markers PAX6 and DCX were increased in BMSCs-mHIF-1 $\alpha$-transplanted rats. These results suggest that HIF-1 $\alpha$ in BMSCs reduces neuronal apoptosis and promotes neurogenesis after stroke in rats.

bone marrow-derived mesenchymal stem cells, hypoxia-inducible factor 1 , stroke, migration

Citation: $\quad$ Ye Z Z, Ye W B, Deng Y B, et al. HIF-1-modified BMSCs improve migration and reduce neuronal apoptosis after stroke in rats. Chin Sci Bull, 2013, 58: 3519-3528, doi: 10.1007/s11434-013-5936-6

As a leading cause of death and disability worldwide, stroke is still an unresolved clinical problem. In experimental stroke, bone marrow-derived mesenchymal stem cells (BMSCs), administered locally or systemically, have been demonstrated to migrate to the ischemic hemisphere, and thereby promote neurologic and functional recovery [1,2]. In vitro study, BMSCs can be induced into both neurons and glia [3]. However, the mechanisms responsible for the therapeutic effects of BMSCs remain unclear. Several factors, such as transdifferentiation, induction of angiogenesis, neurogenesis and activation of endogenous neurorestorative processes, might contribute to the benefits of BMSCs in the ischemic brain [4]. Notably, when $2 \times 10^{6}$ BMSCs are transplanted in the ischemic hemisphere in the range of $0.02 \%$, very rare

$\dagger$ These authors contributed equally to this work.

*Corresponding author (email: dengyub@mail.sysu.edu.cn) grafted BMSCs are observed to express neural markers [2]. In addition, expression of neural markers may not necessarily represent the true neural differentiation and function. Therefore, it appears unlikely that BMSCs would differentiate in situ into the lost neural cells and integrate into neural connections. The more likely mechanism would be that BMSCs provide injured and damaged tissues an "attractive" microenvironment through the secretion of cytokines and trophic factors to activate endogenous brain remodeling. This process may include neurogenesis, angiogenesis, and synaptogenesis, reducing neuronal apoptosis and promoting neuronal proliferation in the boundary zone of the ischemic hemisphere. However, these possible therapeutic potentials remains to be discovered and subsequently further undersood. Many studies suggest genetic modification of BMSCs to improve therapeutic potential of BMSCs treatment on stroke. Indeed, overexpression of trophic factors such as 
BDNF, FGF-2 and HGF in gene-modified BMSCs has been demonstrated to improve neurologic outcomes in animal models of ischemic stroke [5-7].

Hypoxia plays an important role in the pathophysiology of ischemic brain injury. Hypoxia-inducible factor-1 (HIF-1), as a crucial regulator of the cellular response to low oxygen concentration, activates the transcription of a number of genes that code for proteins involving in angiogenesis, glucose metabolism, cell proliferation and migration [8-10]. HIF-1 is a heterodimeric protein that consists of a constitutively expressed HIF-1 $\beta$ subunit and a functional HIF- $1 \alpha$ subunit. Under hypoxic conditions, HIF- $1 \alpha$ degradation is inhibited and dimerizes with the HIF- $\beta$ subunit forming the active HIF-1 complex to activate target genes. The central role of HIF-1 in the cellular response to hypoxia makes this factor an attractive therapeutic target for ischemic stroke. Recently, it is reported that HIF- $1 \alpha$ induced-VEGF overexpression in bone marrow stem cells protect cardiomyocytes against ischemia [11]. A growing body of evidences has shown that HIF-1 stimulates several neurogenic, protective and neurotrophic activities, including proliferation of astrocytes [12] and microglia [13]. In a way, HIF-1 can serve as a neurotrophic, neuroprotective and neuroproliferative factor. This raises the possibility that HIF-1 plays essential roles in BMSC-mediated promotion of neurological and functional recovery in ischemic brain.

In the present study, we intravenously transplanted BMSCs, in which a stable form of HIF- $1 \alpha$ gene was transferred with lentivirus vector $e x$ vivo, into rats with a permanent middle cerebral artery occlusion (MCAO) model. We specifically intended to address the question whether overexpression of HIF-1 $\alpha$ could promote BMSCs therapeutic effect under normoxic and hypoxic conditions. Then investigated whether such a combined therapy could improve the therapeutic effects of BMSCs on ischemia and explored the possible roles of HIF-1 in mediating BMSCs migration, reducing neuronal apoptosis and promoting neurogenesis.

\section{Materials and methods}

\subsection{Animals and the pMCAO Model}

Male healthy Sprague-Dawley rats (weighing 220-250 g) were obtained from the Experimental Animal Center of Sun Yat-Sen University and randomly divided into three groups: the HIF-1-BMSCs $(n=18)$, EGFP-BMSCs $(n=18)$, and the control group $(n=18)$. The animals were kept in a standard light/dark schedule with free access to food and water. All animal procedures were conducted in compliance with the guidelines of the Institutional Animal Care and Use Committee. Permanent middle cerebral artery occlusion was induced by a modification of intraluminal vascular occlusion method as previously described [14,15]. Briefly, the animals were anesthetized with $10 \%$ chloral hydrate $(0.4$ $\mathrm{g} / \mathrm{kg}$, i.p.), the left common carotid artery, external carotid artery (ECA), and internal carotid artery (ICA) were exposed through a ventral midline incision in the neck. A siliconecoated nylon filament at the diameter of $0.234 \mathrm{~mm}$, with its tip rounded by heating near flame, was introduced from the ECA into the lumen of the ICA until it blocked the origin of the MCA.

\subsection{Isolation and culture of BMSCs}

BMSCs were isolated by the density gradient technique as described previously [16]. In brief, the bone marrow was obtained from the femurs and tibias taken from male Sprague-Dawley rats weighting 60-80 g. The bone marrow mononuclear cells were separated over Ficoll-Hypaque gradients (Sigma Chemical Co., St. Louis, MO, USA) at $400 \times g$ for $30 \mathrm{~min}$ and plated into $25 \mathrm{~cm}^{2}$ flasks in Iscove's modified Dulbeccomedium: Nutrient Mixture F-12 (GIBCO, BRL, Grand Island, USA) supplemented with $10 \%$ fetal bovine serum (Kibbutz Beit Haemek, Israel). After the cells were incubated at $37^{\circ} \mathrm{C}$ in $5 \% \mathrm{CO}_{2}$ for $3 \mathrm{~d}$, the culture medium containing non-adherent cells was removed, and the adherent layer was washed once with the fresh medium and then cultured continuously. After the cells reach $80 \%$ confluence, they were incubated at $1: 3$ dilutions. BMSCs were used for intravenous transplantation in these experiments.

\subsection{HIF-1 $\alpha$ mutagenesis}

A full length cDNA of human HIF-1 $\alpha$ was obtained by PCR and cloned in pcDNA3.0 (Invitrogen Corp., Carlsbad, CA, USA), which was used as a template for PCR to induce site-directed mutation at residues P402, P564 and N803 that target HIF- $1 \alpha$ for proteosomal degradation under normoxic conditions [17]. The synthesized primers were: P402A $(\mathrm{CCA} \rightarrow$ GCA), GCTTTAACTTTGCTGGCCGCAGCCGCTGGAGACACA (forward) and TGTGTCTCCAGCGGCTGCGGCCAGCAAAGTTAAGC (reverse); P564G (CCC $\rightarrow$ GGC), CTTGGAGATGTTAGCTGGCTATATCCCAATGGATG (forward) and CATCCATTGGGATATAGCCAGCTAACATCTCCAAG (reverse); N803A (AAT $\rightarrow$ GCT), CCAGTTATGATTGTGAAGTTGCTGCTCCTATACAAGGCAG (forward) and CT GCCTTGTATAGGAGCAGCAACTTCACAATCATAACTGG (reverse). The triple mutations of HIF-1 $\alpha$ cDNA were verified by DNA sequencing.

\subsection{Lentivirus vector construction}

The triple mutant HIF-1 $\alpha$ fragment was sub-cloned into pEGFP-N2 vector (Clontech, Palo Alto, CA, USA) via KpnI and BamHI sites to generate the mHIF-1 $\alpha$-EGFP fusion gene expression plasmid (pEGFP-N2/mHIF-1 $\alpha$ ). The coding sequences of mHIF- $1 \alpha$-EGFP and EGFP were amplified from the above pEGFP-N2/mHIF- $1 \alpha$ plasmid and TOPO cloned into vector $\mathrm{pENTR/D-TOPO}$, and then transferred 
into plenti6/v5-DEST by using the Gateway LR recombination reaction (Invitrogen). Lentiviral expression plasmids containing mHIF-1 $\alpha$-EGFP sequence and EGFP sequence were termed Lv-mHIF-1 $\alpha$-EGFP and Lv-EGFP, respectively. The integrity of plasmids were identified by restriction enzyme analysis and confirmed by DNA sequencing.

\subsection{Lentivirus production and infection of BMSCs}

Recombinant lentiviruses were produced by cotransfection of 293 T cells with the packaging plasmids (pLP1, pLP2, and $\mathrm{pLP} / \mathrm{VSVG}$ ) and lentivirus expression plasmids (LvmHIF-1 $\alpha$-EGFP or Lv-EGFP), using the Lipofectamine 2000 reagent (Invitrogen). The virvus-containing supernatant was harvested $48 \mathrm{~h}$ posttransfection and filtered through a Millex HV filter $(0.45 \mu \mathrm{m}$, Millipore Corp). BMSCs were infected with the lentivirus in the presence of $5 \mu \mathrm{g} / \mathrm{mL}$ Polybrene (Sigma-Aldrich, Steinheim, Germany). Stable transfectants were selected in $50 \mu \mathrm{g} / \mathrm{mL}$ Blasticidin for $14 \mathrm{~d}$. BMSCs infected with Lv-HIF-1 $\alpha$-EGFP were named BMSCsmHIF- $1 \alpha$, and BMSCs infected with Lv-EGFP were named BMSCs-EGFP.

\subsection{Behavioral tests}

All animals were received a modified neurological severity score (mNSS) tests before MCAO and 1, 7, 14 and $28 \mathrm{~d}$ post MCAO ( $n=6$ for each group). Neurological function was graded on a scale of $0-18$, with normal score as 0 and maximal deficit score 18 . In the severity scores of injury, the higher the score, the more severe the neurological deficit. Spatial learning and memory was assessed using a modified version of the Morris water-maze test [18]. Briefly, the rats were placed in a circular tank of warm, opaque water with a hidden platform. During training trials, latency to find the platform location is recorded. During probe trials, the platform is removed, and the percentage of time spent in the quadrant that normally contains the platform is compared to the time spent in other quadrants.

\subsection{Intravenous administration of BMSCs}

In vivo study, the experimental groups consisted of control group: rats were given MCAO without cell administration but with $1 \mathrm{~mL}$ PBS; BMSCs-EGFP group: rats were transplanted with $2 \times 10^{6}$ BMSCs-EGFP; BMSCs-mHIF- $1 \alpha$ group: rats received BMSCs-mHIF- $1 \alpha\left(2 \times 10^{6}\right.$ cells $)$ via intravenously injection at $3 \mathrm{~h}$ after MCAO. All the transplantation procedures were performed under aseptic conditions.

\subsection{Cerebral infarct measurement}

Cerebral infarct volume was measured by staining brain slices with triphenyltetrazolium chloride (TTC; Sigma, St. Louis, MO, USA) $7 \mathrm{~d}$ after MCAO. The animals $(n=3$ for each group) were decapitated under deep anesthesia with $10 \%$ chloral hydrate $(0.8 \mathrm{~g} / \mathrm{kg}$, i.p. $)$ The brains were quickly removed and sliced into 5 coronal sections at 3-mm intervals. The slices were incubated in a $2 \%$ solution of TTC at $37^{\circ} \mathrm{C}$ for $30 \mathrm{~min}$ and then fixed in $10 \%$ formaldehyde solution overnight. The infarct area of each brain slice was analyzed using the Image-Pro plus 5.0 analysis software. The total infarct volume for each brain was calculated by summation of the infarct area of all brain slices.

\subsection{Semi-quantitative RT-PCR}

Total RNA was extracted from BMSCs and brain biopsy tissues using TRIzol reagent (Invitrogen) according to the manufacturer's protocol. The first strand of cDNA was obtained using Revert Aid First Strand cDNA Synthesis Kit (MBI, Norwalk, CT, USA). PCR was performed with $r T a q$ polymerase (TaKaRa, Japan). The cycling conditions used were: denaturing at $94^{\circ} \mathrm{C}$ for $5 \mathrm{~min}$, annealing at $60^{\circ} \mathrm{C}$ for 1 min, and polymerization at $72^{\circ} \mathrm{C}$ for $1 \mathrm{~min}$. The resulting PCR products were analyzed by $1.5 \%$ agarose electrophoretic gels using DL2000 DNA Marker (TaKaRa) as a size reference. Gel images were obtained and the densities were quantified with the application Bio-ID gel analysis software (Vilber Lourmat, Marne-la-Vallée, France). All results represented the average density of positive bands obtained from at least three independent experiments.

\subsection{Western blot}

Fresh tissue samples of ischemic hemisphere, obtained at $7 \mathrm{~d}$ after MCAO, were homogenized for western blot study. After lysis and evaluation of protein content via Bradford protein assay, equal amounts of protein were separated by SDS-PAGE and transferred to PVDF membrane $(0.45-\mu \mathrm{m}$ pore size, Roche Molecular Biochemicals, South San Francisco, CA, USA). The blocked membranes were then incubated with different antibodies overnight at $4{ }^{\circ} \mathrm{C}$ : mouse anti-HIF-1 (1:1000; Chemicon). After washing with TBST, the membranes were incubated with HRP-conjugated antibody $\left(1: 2000\right.$; Amersham) for $60 \mathrm{~min}$ at $37^{\circ} \mathrm{C}$, and then detected with ECL (Amersham). The optical density of the bands was quantified by Bio-Rad Image analysis.

\subsection{TUNEL}

DNA strand breaks were detected using TUNEL method. Briefly, brain sections were deparaffinized and digested with proteinase K (Roche) and then covered with TUNEL reaction mixture, followed by converter-POD/wash buffer. Finally, sections were covered with AEC substrate solution and detected with diaminobenzidine (DAB, Chemicon ES005$10 \mathrm{ML}$ ). The integrated optical density (IOD) of apoptotic neuronal cells was measured in the cortex and hippocampus of ipsilateral hemisphere using light microscope (Olympus 
BH2-RFL-T3 Japan 400x) and color image recorder (JVC KY F30B 3-CCD Germany) and analyzed using Pro Plus software 5.1 (Media Cybernetics, Bethesda, MD, USA) by the protocol. The neuronal apoptosis from at least three sections per animal selected randomly was evaluated immunohistochemically to determine IOD of the apoptotic cells exhibiting positive staining. For each slide, 6 fields were selected and examined randomly, in a defined rectangular field area ( $\times 200$ objective), a total of at least 2000 cells per field were counted. The apoptotic index was determined (i.e., IOD of apoptotic cells divided bythe total neuronal cells counted $\times 200$ ) from a total of 18 fields per brain. The assays were performed in a double-blinded manner.

\subsection{Immunofluorescence}

Immunofluorescent evaluation was performed on rat brain samples collected at day 1, 7, 14, following the intravenous transplantation of BMSCs into rats subjected to MCAO. For double fluorescence labeling of Pax6/DCX in the hippocampus region at day 7 and cortex at day 14 post MACO, respectively. Sections were incubated with primary antibodies: mouse anti-Pax6 (1:300; Chemicon); goat anti-DCX (1:400) (Santa Cruz, CA, USA), respectively. After washing with PBS, the sections were incubated with the appropriate fluorescent secondary antibodies for $1 \mathrm{~h}$ at $37^{\circ} \mathrm{C}$. Negative control sections from each animal received identical preparations for immunofluorescence staining, except that the primary antibodies were omitted. The sections were examined with an IX71 SIF-2 fluorescence microscope for fluorescence equipped with an Olympus digital camera/ Image-Pro plus software 5.1. For each section, six microscope fields in a defined rectangular field area $(\times 200$ objective) were selected and analyzed randomly. Cells were counted in images captured from these areas.

\subsection{Statistical analysis}

The measurements were performed blindly. SigmaStat (SPSS, Inc., Chicago, Illinois, USA) statistical software was used for all statistical analysis. All data represented at least three independent experiments and statistical differences between the means for the different groups were evaluated with a oneway analysis of variance (ANOVA). Results are expressed as mean $\pm \mathrm{SD} . P<0.05$ was set as statistical significance.

\section{Results}

\subsection{Lentivirus-mediated constitutive expression of HIF- $1 \alpha$ in BMSCs}

In order to induce stable expression of HIF-1 $\alpha$, BMSCs were infected with lentivirus encoding the triple mutant form HIF- $1 \alpha$, in which site-directed mutation at residues P402, P564 and N803 leads to stabilization of HIF-1 $\alpha$ in normoxia (Figure 1(a)). Semi-quantitative RT-PCR showed
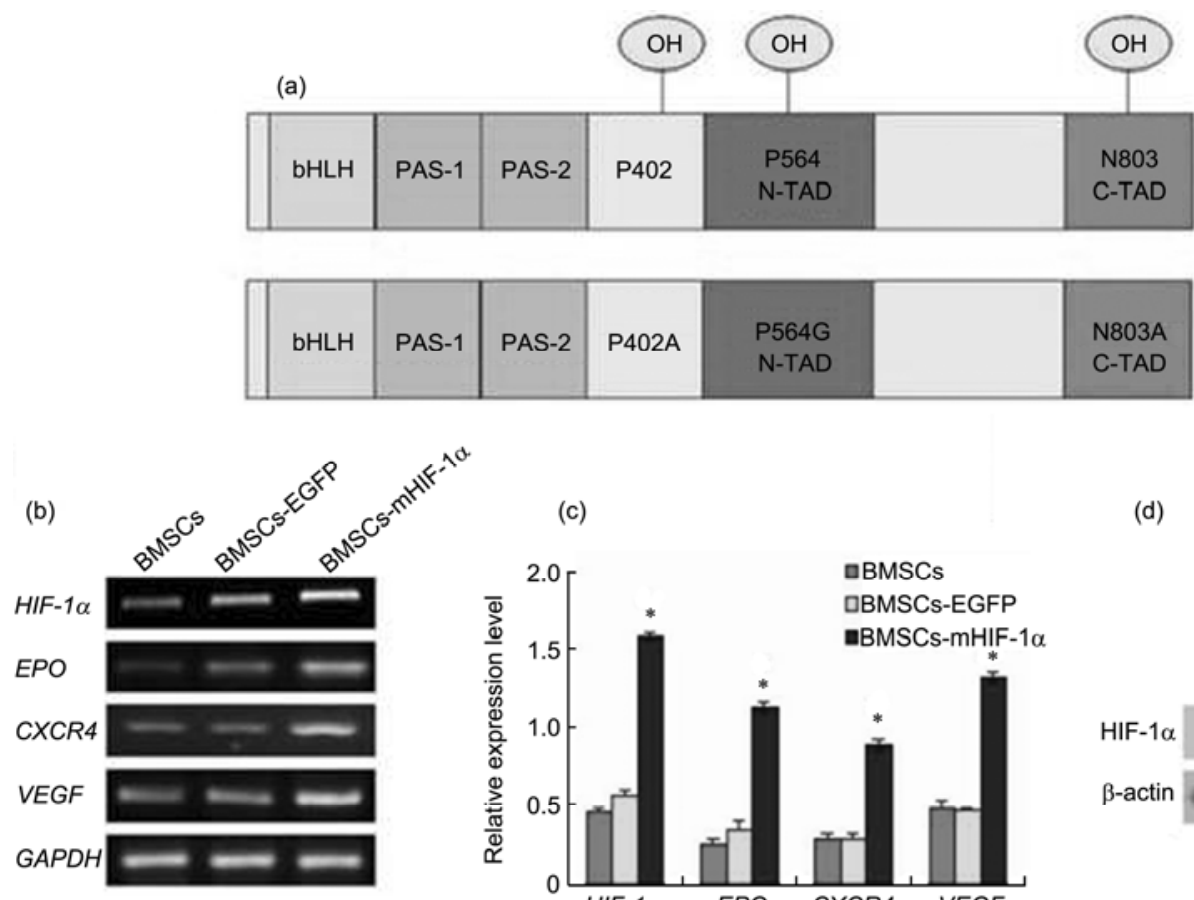

(c)

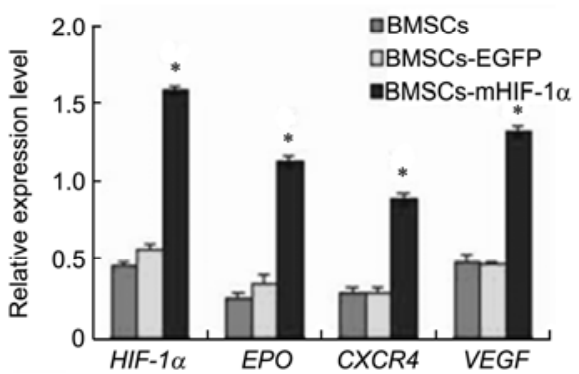

(d)

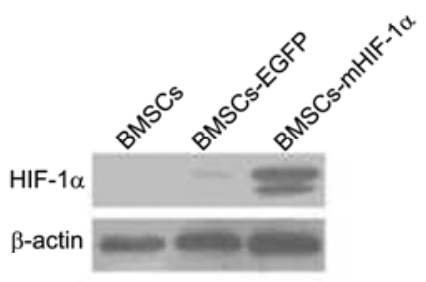

Figure 1 Lentivirus-mediated constitutive expression of HIF-1 $\alpha$ in BMSCs. (a) Aschematic illustrating the location of the three point mutations in relation to the various domains of the HIF-1 $\alpha$ triple mutant protein. (b) RT-PCR analysis of mRNA isolated from BMSCs $48 \mathrm{~h}$ post-transfection BMSCs-mHIF-1 $\alpha$ group or BMSCs-EGFP. (c) Statistical analysis of RT-PCR. (d) Western blot analysis of protein isolated from BMSCs $48 \mathrm{~h}$ post-transfection BMSCsmHIF-1 $\alpha$ group BMSCs-EGFP or control. * indicates $P<0.05$. 
that the expression level of $H I F-1 \alpha$ mRNA was strongly induced in BMSCs-mHIF-1 $\alpha$. Interestingly, the mRNA level of the HIF- $1 \alpha$ target genes VEGF, EPO and CXCR4 were also found significantly increased (Figure 1(b)(c)). Western blot analysis further confirmed a strong up-regulation of HIF- $1 \alpha$ in BMSCs-mHIF-1 $\alpha$ (Figure 1(d)). Even the cells were cultured in nomoxia, HIF-1 $\alpha$ was hardly detectable in non-transduced cells.

\subsection{Neurologic outcome}

Only moderate and severely injured rats $(\mathrm{mNSS}>6)$ were included in subsequent experiments $(>90 \%$ of rats in each group). No significant difference was detected among all the groups prior to surgery and before treatment. The rats treated with BMSCs-mHIF- $1 \alpha$ had significant functional recovery on mNSS when compared with the control group rats from day 7 after MCAO. However, the significant neurological functional recovery was observed in the BMSCsEGFP group 1 compared with the sham-group rats $14 \mathrm{~d}$ after MCAO. When time was going by day 14 and 28, the significant recovery in BMSCs-mHIF- $1 \alpha$ was observed when compared with either the BMSCs-EGFP or the control groups (Figure 2). Furthermore, as tested in the Morris WaterMaze test at both day 14 and 28 post MCAO, the latency periods and travel distances in two BMSC-treated group rats were all significantly shorter than those in the control group (Table 1). Under these circumstances, there was no significant difference observed between the two BMCS-treated groups.

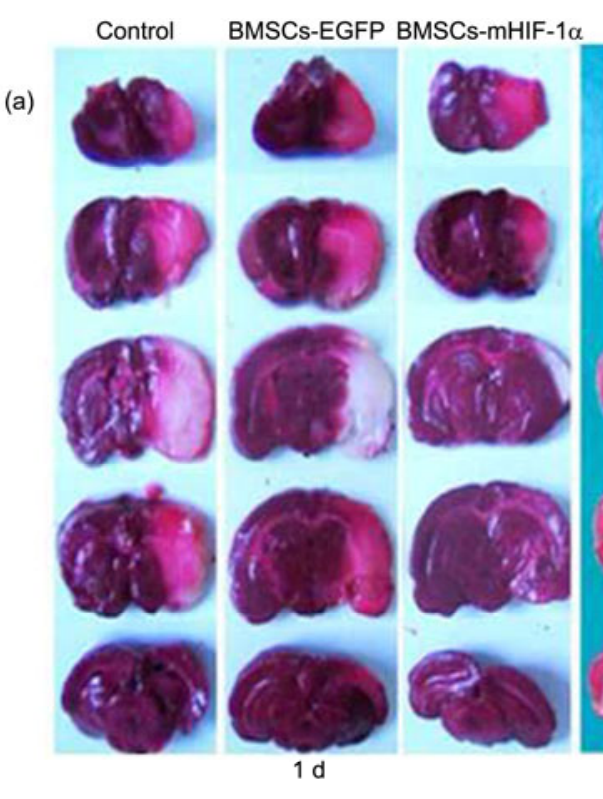

(b)

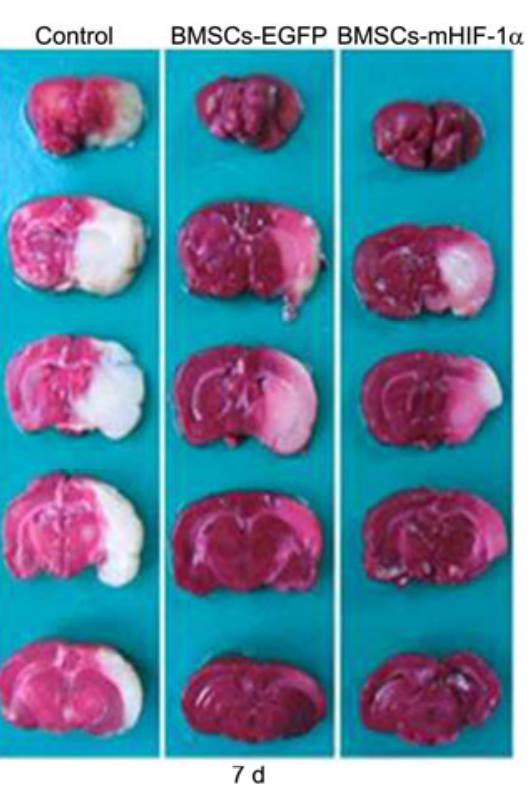

$7 \mathrm{~d}$

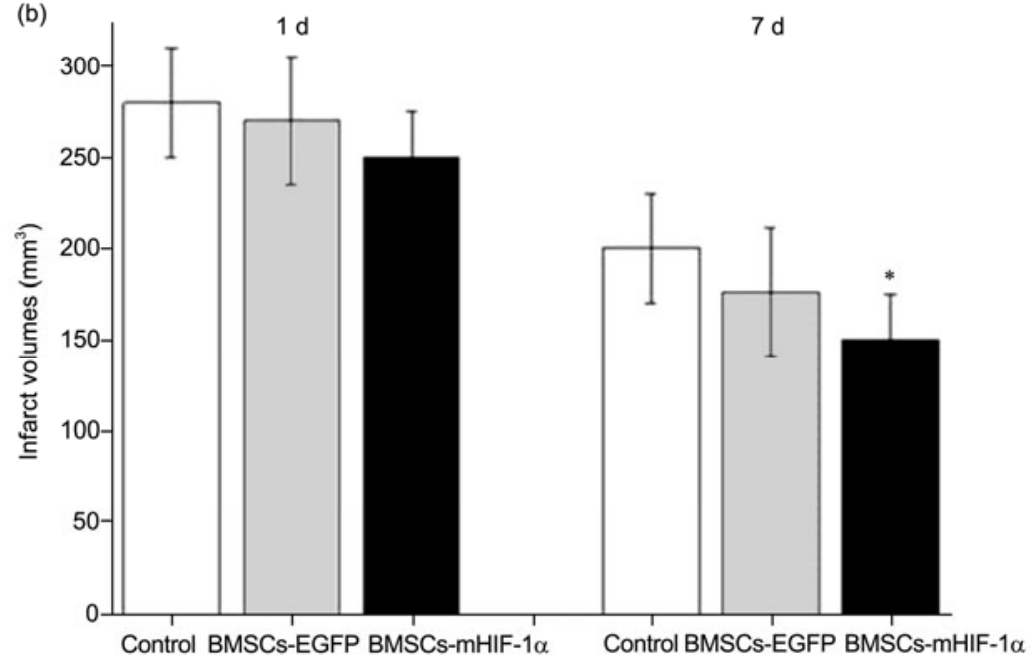

Figure 2 Cerebral infarct volumes of rats 7 days after MCAO. (a) TTC staining of brain slices. (b) A tendency of infarct volume decreased in HIF-1 $\alpha$ BMSCs-treated rats was noticed. Significant reductions of infarct volume in BMSCs-mHIF-1 $\alpha$ group rats were observed when compared with the control or the BMSCs-EGFP group $(* P<0.05)$. 


\subsection{Quantitative analysis of infarct volume}

Infarct volumes were determined from TTC-stained sequential coronal sections of experimental rats. While there was a tendency for infarct volume to decrease in the BMSCsmHIF- $1 \alpha$ group as detected $1 \mathrm{~d}$ after MCAO, this difference was not statistically significant. At day 7 , there were significant reductions of infarct volume in rats of the BMSCsmHIF-1 $\alpha$ group compared with the BMSCs-EGFP group and the control group, respectively (Figure 2).

\subsection{Migration of transplanted BMSCs in ischemic brain}

Immunofluorescent evaluation was performed on rat brain samples collected at day $1,7,14$, following the intravenous transplantation of BMSCs into rats subjected to MCAO. GFP-labeling of BMSCs facilitated tracking the transplanted cells by fluorescent microscopy. One day after cell infusion, the majority of BMSCs were initially localized within the olfactory area surrounding the occlusion point of the middle cerebral artery, which could be defined as the ischemic core. At day 7 after transplantation, BMSCs were found to have entered the boundary area of the injured cortex, mainly including hypothalamus, thalamus and internal ventricle. At later time points of day 14, the majority of transplanted cells were distributed in the striatum and motor cortex fields adjacent to the infarct region (Figure 3).

\section{5 mHIF-1 $\alpha$-transduced BMSCs reduce neuronal apoptosis in ischemic tissue}

Overall, many apoptotic neuronal cells could be detected by TUNEL staining in cortex and hippocampus of all experimental rats $1 \mathrm{~d}$ after MCAO. However, the number of apoptotic cells $(P<0.01)$ in hippocampus of the BMSCs-mHIF-1 $\alpha$ rats were found significantly less than that of the other two groups at day 7 after MCAO (Figure 4).

\section{6 mHIF-1 $\alpha$-Transduced BMSCs induced neurogenesis in ischemic tissue}

Doublecortin (DCX) is an important neuronal microtubuleassociated protein, which can be used as the migration marker of immature neurons. Individual adult neural stem cells can

Table 1 The comparison of acquisition performance on the Morris Water-Maze test among the three group rats $(n=6)^{\text {a) }}$

\begin{tabular}{|c|c|c|c|c|c|c|}
\hline \multirow{2}{*}{ Time (d) } & \multicolumn{2}{|c|}{ Control } & \multicolumn{2}{|c|}{ BMSCs-EGFP } & \multicolumn{2}{|c|}{ BMSCs-mHIF- $1 \alpha$} \\
\hline & Escape latency (s) & Path length $(\mathrm{cm})$ & Escape latency (s) & Path length $(\mathrm{cm})$ & Escape latency (s) & Path length $(\mathrm{cm})$ \\
\hline 14 & $101.5 \pm 10.6^{*}$ & $1872.3 \pm 68.6^{*}$ & $35.8 \pm 5.9$ & $612.1 \pm 33.4$ & $25.3 \pm 3.1$ & $523 \pm 23.6$ \\
\hline
\end{tabular}

a) The above escape latency $(\mathrm{s})$ and path length $(\mathrm{cm})$ of rats in the Morris Water-Maze indicated that ischemia-induced spatial learning and memory deficits were significantly ameliorated in both BMSCs-mHIF-1 $\alpha$ group and BMSCs-EGFP group compared with control group at 14 and $28 \mathrm{~d}$ after transplantation. Values are mean $\pm \mathrm{SD}$, and analyzed with one-way ANOVA. $* P<0.05$.

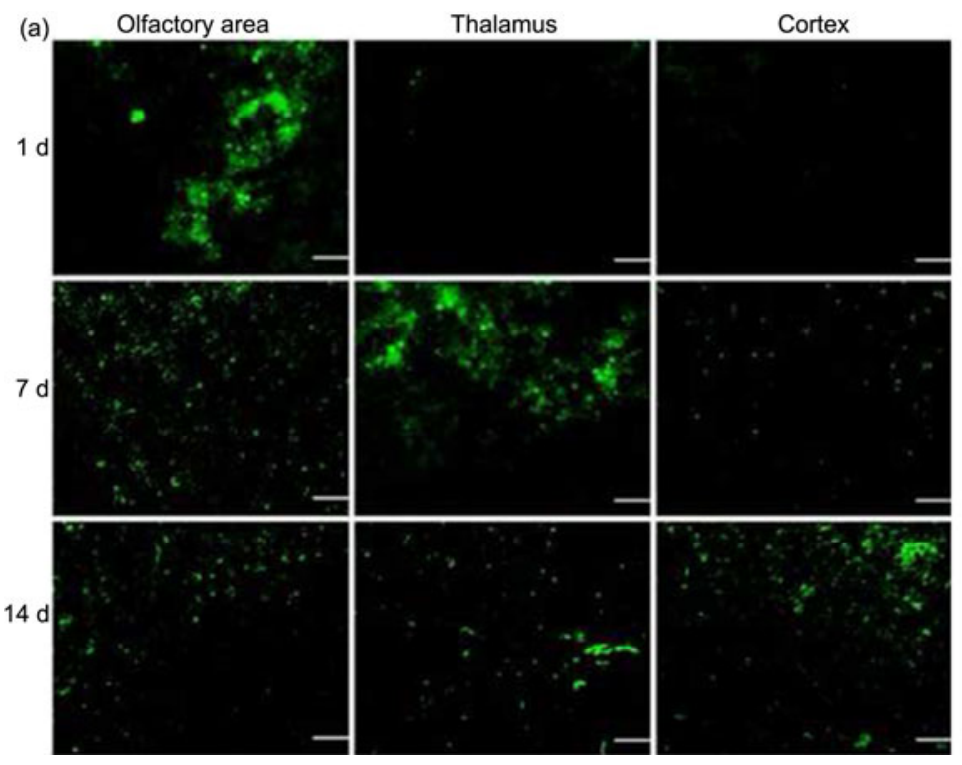

(b)

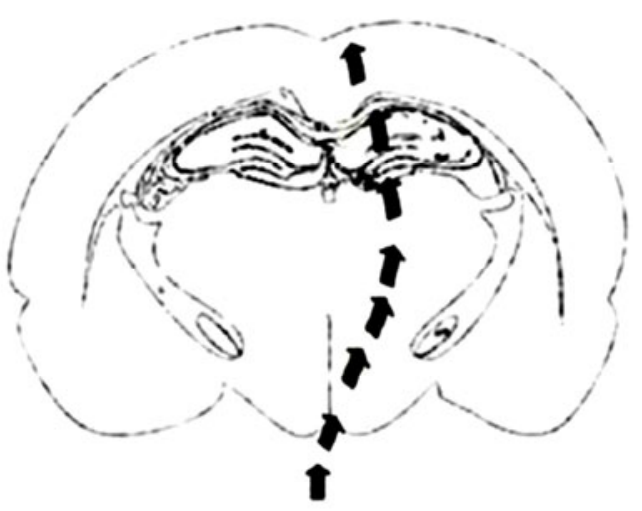

Figure 3 Presence of GFP+ cells in the rat brain at different time points post MCAO. (a) At day 1, the majority of GFP+ cells were confined to the olfactory area, belonging to the infarct core. At day 7, abundant GFP+ cells are detected in the boundary area of the injured cortex, which mainly includes hypothalamus, thalamus and internal ventricle. At day 14, the majority of GFP+ cells were distributed in the striatum and motor cortex fields adjacent to the infarct region. (b) The GFP+ cell distribution delineated by arrow suggesting the migrate route of transplanted BMSCs. Scale bar, $100 \mu \mathrm{m}$. 
highly express pax6, Emx2, and Mash1 transcription factors, which can also be identified different subtypes of neural progenitor cells. Pax6/DCX positive cells indicate neuroblast. Immunohistochemitry with two monoclonal antibodies specific to immature neuron (pax6 and DCX) were employed to identify the endogenous neural stem cells (NSCs). It was demonstrated that the pax6/DCX double positive cells were mainly present in hippocampus and penumbral regions of cortex. The number of pax6/DCX cells was accounted in six randomly selected locations of cortex and hippocampus. A significant increase of pax6/DCX cells in hippocampus was found in the BMSCs-mHIF-1 $\alpha$ group at day 7 post MCAO (Figure 5(a)). Meanwhile, the higher number of pax6/DCX cells were also found in cortex of BMSCs-mHIF- $1 \alpha$ group rats at day 14 after MCAO (Figure $5(\mathrm{~b})$ ). These results indicated that the mHIF-1 $\alpha$-transduced BMSCs injection enhanced the neuronal proliferation in the hippocampus and cortex post MCAO.

\section{Discussion}

Stroke is characterized by the interruption of blood flow to the brain, which initiates a series of complex events that cause neuronal death and neurological deficits. Recent studies have demonstrated that neurogenesis occurs throughout adulthood and that neural stem cells reside in the adult central nervous system (CNS) [19]. The survival, proliferation, migration and differentiation of neural stem cells are regulated by a variety of endogenous and exogenous factors, including oxygen concentration [20], growth factors [21-24] and cytokines [25,26]. Some of these factors have been confirmed to guide stem cells to the state where they can improve the functional recovery of brain tissue after stroke [27]. Meanwhile, intravenous administration of BMSC has been demonstrated to improve neurological deficits and accelerate their recovery in a rat model of stroke. Application of BMSCs in clinical trials further showed positive results and no side effects were detected. This study provides additional supportive evidences to the earlier conclusion that administration of BMSCs could significantly promote morphological and functional recovery. Although the therapeutic mechanisms remained to be clarified, induction of neurogenesis by means of transplanting BMSCs in ischemic brain may contribute to functional improvement of brain, as it has been suggested that BMSCs may stimulate the adjacent host

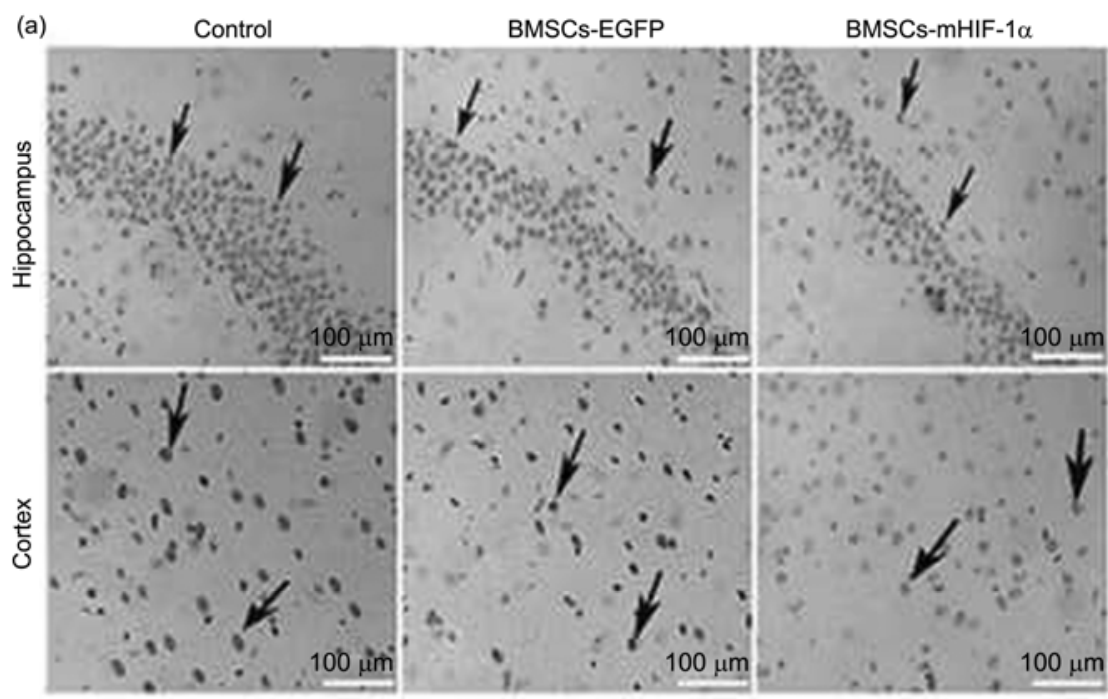

(b)

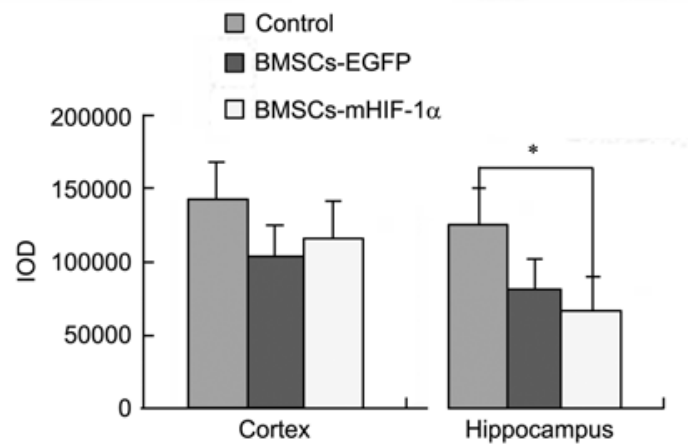

Figure 4 Apoptotic cells present in rat brain as detected by TUNEL-staining. (a) TUNAL-staining at day7 post MCAO, respectively. (b) Photographs for cortex and hippocampus tissues of TUNEL-staining and the lower panels show the counting of TUNEL-stained cells. $* P<0.01$. 


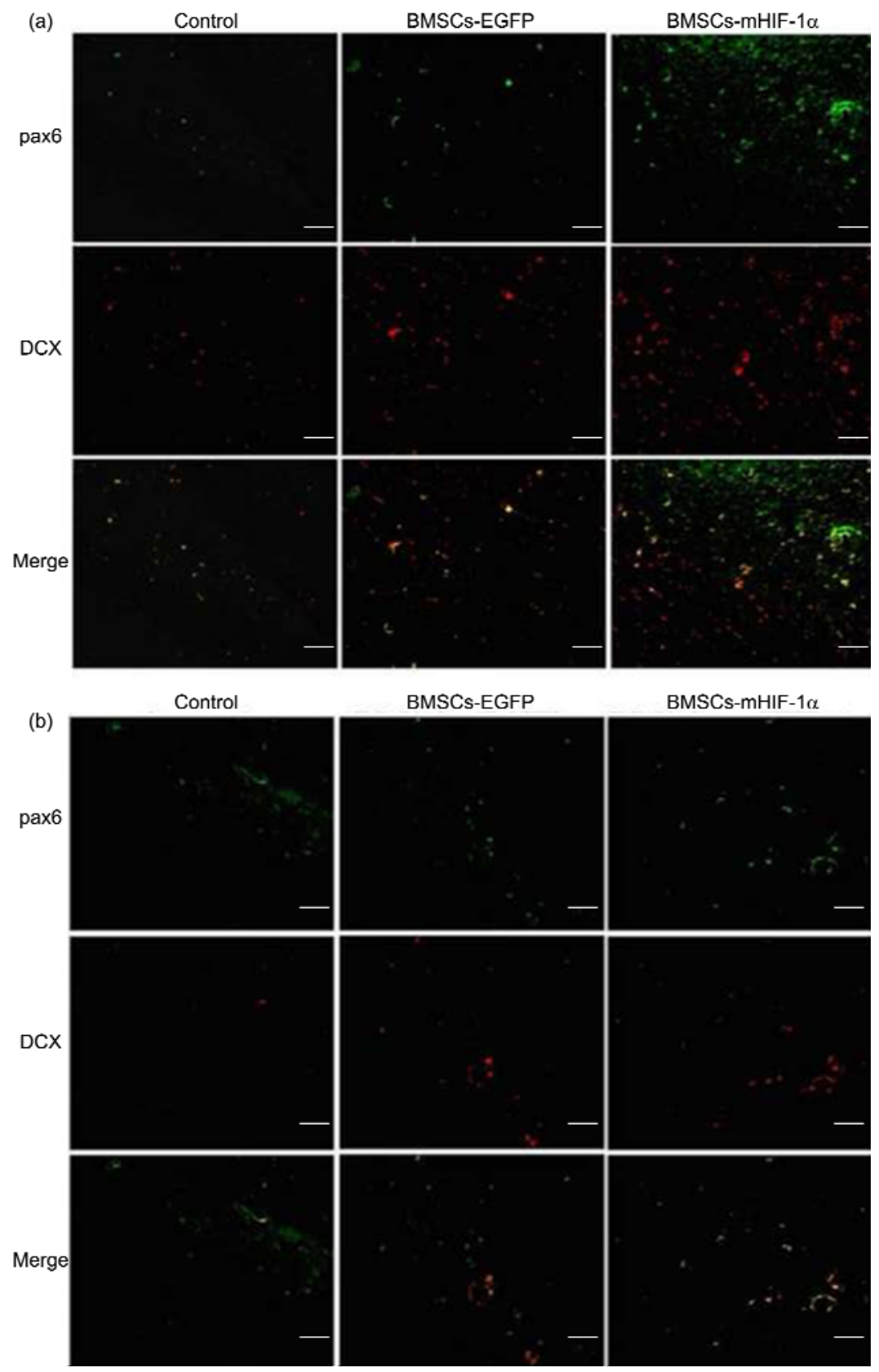

Figure 5 Pax6 and DCX expression in the ischemic tissues of rat brain. Double immunofluorescent staining in the hippocampus region at day 7 (a) and cortex at day 14 (b) post MACO, respectively. In both the shown tissue regions, a significant increase of pax6/DCX double positive cells was observed in BMSCs-mHIF- $1 \alpha$ group rats at $7 \mathrm{~d}$ after MCAO $(* P<0.05)$. Scale bar, $100 \mu \mathrm{m}$.

cells by producing trophic, proangiogenic and other paracrine factors.

Recent studies have shown that overexpression of HIF-1 could drive a potent paracrine activity and subsequently elevate the levels of growth factors and cytokines [28-30], which suggested that HIF-1 may be one of the potential regulators for the treatment of brain ischemia. In our study, intravenous administration of BMSCs-mHIF- $1 \alpha$ could improve the neurological restoration and significantly reduce the infarction volume in a rat model of stroke. Furthermore, constitutive expression of HIF-1 $\alpha$ could enhance the migra- tion of BMSCs toward ischemic cerebral hemisphere, reduce neuronal apoptosis and promote neuronal proliferation. Although how the constitutive expression of HIF- $1 \alpha$ act on BMSCs remains unknown, the result that expression of the four HIF-1 target genes, including EPO, VEGF and CXCR4, were upregulated in our study do bring us some clues to answer these questions. Therefore, the positive roles of HIF-1 in the regeneration of brain tissue might be via these molecules.

Currently the roles of HIF-1 in the progression of cerebral ischemia appear controversial [31,32]. A number of previous studies clearly indicated that activation of HIF-1 is 
a central part of adaptive responses to attenuate the harmful effects of ischemic brain injury. Particularly, HIF-1 $\alpha$ upregulation by hypoxia pre-conditioning has been shown to be neuroprotective against cerebral ischemia [32,33]. HIF-1 regulates the transcription of many well-established neuroprotective genes in response to hypoxia, which is of crucial importance in governing essential steps of neurogenesis, including cell survival, migration, proliferation and differentiation. In normoxia, HIF- $1 \alpha$ is hydroxylated on Pro-402 and Pro-564 in the oxygen-dependent degradation domain (ODD) by EGLN1/PHD1 and EGLN2/PHD2. The hydroxylated prolines promote interaction with VHL, initiating rapid ubiquitination and subsequent proteasomal degradation. This hydroxylation is inhibited by the $\mathrm{Cu} / \mathrm{Zn}$ chelatorClioquinol. S-nitrosylation of Cys-800 may be responsible for increased recruitment of p300 coactivator necessary for transcriptional activity of HIF-1 complex requiring phosphorylation for DNA binding. HIF-1 transcription, as a primary determinant of embryonic morphogenesis, is essential for normal brain development [34,35]. Targeted disruption of HIF-1 results in severe cardiac and vascular malformation leading to embryonic lethality by day 10.5 of gestation [8]. Conditional inactivation of HIF-1 $\alpha$ gene in midbrain-derived neural precursor cells (mNPC) indicates that HIF- $1 \alpha$ is required for the proliferation and survival of precursor cells. These studies support the conclusion that HIF-1 has beneficial roles in neuroprotection. However, some other studies suggest that activation of HIF-1 signaling may have a harmful effect on ischemic brain [36-38]. In vivo HIF-1 $\alpha$ knockout studies showed that deletion of HIF-1 in the brain led to an amelioration of apoptosis in the nervous system of the adult mouse [38]. HIF-1 $\alpha$ inhibition after cerebral ischemia showed that decreasing the expression of HIF-1 $\alpha$ might be neuroprotective [36,37]. Therefore, clarification of the detailed molecular and cellular mechanisms of HIF in neuroprotection need further study. One of the possible explanations may be that the effects of HIF-1 $\alpha$ activation on ischemic brain depend on the cell types [12] and the duration [39]. In our present study, we demonstrated that constitutive expression of HIF-1 in BMSCs played positive roles, including improving cell migration, reducing neuronal apoptosis and promoting neuronal proliferation. Our results are well consistent with many drawn from previous reports. HIF-1 $\alpha$ mediates neural progenitor cell proliferation and differentiation [40,41]. More importantly, significant improvement in neurologic function of ischemic brains was further observable, which might be due to the constitutive expression of HIF-1 in transplanted BMSCs.

Various molecular factors including chemokines, cytokines, matrix molecules, together with their specific receptors, may contribute to cell migration [42].The enhanced ability of mHIF-1-modulated BMSCs to migrate to the sites of damage was demonstrated in the present study. Among multiple potential factors, the SDF-1/CXCR4 axis has been reported to be expressed in a wide range of tissues, including brain, heart, kidney, liver, lung, and spleen [43], which has been involved in several diseases such as ischemic cardiomyopathy and brain disorders [44]. Previous studies have demonstrated that HIF-1 can induce SDF-1 expression and result in enhancing adhesion, migration and homing of circulating CXCR4-positive progenitor cells to ischemic tissue. Blockade of SDF-1 in ischemic tissue or CXCR4 on circulating cells prevents progenitor cell recruitment to the sites of injury [45]. Meanwhile, CXCR4, also a HIF-1-target gene, was up-regulated in mHIF- $1 \alpha-B M S C s$ and may possibly contribute to the enhanced migration of these cells.

The hyperexpression of HIF-1 $\alpha$ protein has been shown to upregulate downstream gene expression even under conditions of normoxia [46]. Our data showed that BMSCsmHIF-1 $\alpha$ significantly promoted the expressions of EPO and VEGF, which may subsequently provide an appropriate microenvironment for the damaged neuronal cells to neurogenesis. In addition, MSCs within the ischemic brain can induce the production of growth factors within the parenchymal tissue, particularly from astrocytes, as suggested in a co-culture system of human NSCs with astrocytes, where the signal transduction cascades of cultured astrocytes can be altered by MSCs. Astrocytes can also express angiopoietin1 and its receptor Tie2, which both can contribute to both angiogenesis and the maturation of newly formed vessels. Furthermore, MSCs can also induce additional proangiogenic factors and trophic factors by endogenous endothelial cells. The new and activated vessels then likely orchestrate further angiogenesis, attract neuroblasts to their locale, and enhance white matter and neurite plasticity. The neuroblasts, which are attracted to vessels, also subsequently further amplify the angiogenic response, leading to recovery.

Taken together, our study and others demonstrated that the constitutive expression of HIF-1 in BMSCs improve their migration toward ischemic cerebral hemisphere, reduce neuronal apoptosis and promote neuronal proliferation after stroke in rats.

This work was supported by the National Natural Science Foundation of China (30901547/C160803), the Ministry of Education of Guangdong Province University-Industry Cooperation Project (2012B091100457), the Science and Technology Plan Project of Guangdong Province (2012B091100457) and the Natural Science Foundation of Guangdong Province (9151503102000023).

1 Brazelton T R, Rossi F M, Keshet G I, et al. From marrow to brain: 34 expression of neuronal phenotypes in adult mice. Science, 2000, 290: 1775-1779

2 Chen $\mathrm{X}, \mathrm{Li} \mathrm{Y}$, Wang $\mathrm{L}$, et al. Ischemic rat brain extracts induce human marrow stromal cell growth factor production. Neuropathology, 2002, 22: 275-279

3 Yang S H, Gao Q, Bao L H, et al. Striatal extracts promote the dopaminergic differentiation of GFP-bone mesenchymal stem cells. Neurosci Lett, 2012, 2: 115-120

4 Dharmasaroja P. Bone marrow-derived mesenchymal stem cells for the treatment of ischemic stroke. J Clin Neurosci, 2009, 16: 12-20

5 Ikeda N, Nonoguchi N, Zhao M Z, et al. Bone marrow stromal cells 
that enhanced fibroblast growth factor- 2 secretion by herpes simplex virus vector improve neurological outcome after transient focal cerebral ischemia in rats. Stroke, 2005, 36: 2725-2730

6 Kurozumi K, Nakamura K, Tamiya T, et al. BDNF gene-modified mesenchymal stem cells promote functional recovery and reduce infarct size in the rat middle cerebral artery occlusion model. Mol Ther, 2004, 9: 189-197

7 Zhao M Z, Nonoguchi N, Ikeda N, et al. Novel therapeutic strategy for stroke in rats by bone marrow stromal cells and ex vivo HGF gene transfer with HSV-1 vector. J Cereb Blood Flow Metab, 2006, 26: $1176-1188$

8 Iyer N V, Leung S W, Semenza G L. The human hypoxia-inducible factor $1 \alpha$ gene: HIF1A structure and evolutionary conservation. Genomics, 1998, 52: 159-165

9 Lee K A, Roth R A, LaPres J J. Hypoxia, drug therapy and toxicity. Pharmacol Ther, 2007, 113: 229-246

10 Swanson R A, Morton M T, Tsao-Wu G, et al. A semiautomated method for measuring brain infarct volume. J Cereb Blood Flow Metab, 1990, 10: 290-293

11 Dai Y, Xu M F, Wang Y G, et al. HIF-1 $\alpha$ induced-VEGF overexpression in bone marrow stem cells protects cardiomyocytes against ischemia. J Mol Cell Cardiol, 2007, 42: 1036-1044

12 Vangeison G, Carr D, Federoff H J, et al. The good, the bad, and the cell type-specific roles of hypoxia inducible factor-1 alpha in neurons and astrocytes. J Neurosci, 2008, 28: 1988-1993

13 Belayev L, Alonso O F, Busto R, et al. Middle cerebral artery occlusion in the rat by intraluminal suture neurological and pathological evaluation of an improved model. Stroke, 1996, 27: 1616-1622

14 Longa E Z, Weinstein P R, Carlson S, et al. Reversible middle cerebral artery occlusion without craniectomy in rats. Stroke, 1989, 21: 89-91

15 Lu D Y, Liou H C, Tang C H, et al. Hypoxia-induced iNOS expression in microglia is regulated by the PI3-kinase/Akt/mTOR signaling pathway and activation of hypoxia inducible factor-1alpha. Biochem Pharmacol, 2006, 72: 992-1000

16 Colter D C, Sekiya I, Prockop D J. Identification of a subpopulation of rapidly self-renewing and multipotential adult stem cells in colonies of human marrow stromal cells. Proc Natl Acad Sci USA, 2001, 98: 7841-7845

17 Masson N, Willam C, Maxwell P H, et al. Independent function of two destruction domains in hypoxia-inducible factor- $\alpha$ chains activated by prolyl hydroxylation. EMBO J, 2001, 20: 5197-5206

18 Puurunen K, Jolkkonen J, Sirviö J, et al. Selegiline combined with enriched-environment housing attenuates spatial learning deficits following focal cerebral ischemia in rats. Exp Neurol, 2001, 167: 348-355

19 Reynolds B A, Weiss S. Generation of neurons and astrocytes from isolated cells of the adult mammalian central nervous system. Science, 1992, 255: 1707-1710

20 Horie N, So K, Moriya T, et al. Effects of oxygen concentration on the proliferation and differentiation of mouse neural stem cells in vitro. Cell Mol Neurobiol, 2008, 28: 833-845

21 Felling R J, Levison S W. Enhanced following stroke. J Neurosci Res, 2003, 73: 277-283

22 Forsberg-Nilsson K, Behar T N, Afrakhte M, et al. Platelet-derived growth factor induces chemotaxis of neuroepithelial stem cells. J Neurosci Res, 1998, 53: 521-530

23 Johe K K, Hazel T G, Muller T, et al. Single factors direct the differentiation of stem cells from the fetal and adult central nervous system. Genes Dev, 1996, 10: 3129-3140

24 Vicario-Abejon C, Johe K K, Hazel T G, et al. Functions of basic fibroblast growth factor and neurotrophins in the differentiation of hippocampal neurons. Neuron, 1995, 15: 105-114

25 Cameron H A, Hazel T G, McKay R D. Regulation of neurogenesis by growth factors and neurotransmitters. J Neurobiol, 1998, 36: 287-306

26 Shingo T, Sorokan S T, Shimazaki T, et al. Erythropoietin regulates the in vitro and in vivo production of neuronal progenitors by mam- malian forebrain neural stem cells. J Neurosci, 2001, 21: 9733-9743

27 Leker R R. Manipulation of endogenous neural stem cells following ischemic brain injury. Pathophysiol Haemost Thromb, 2006, 35: 58-62

28 Ben-Shoshan J, Schwartz S, Luboshits G, et al. Constitutive expression of HIF- $1 \alpha$ and HIF- $2 \alpha$ in bone marrow stromal cells differentially promotes their proangiogenic properties. Stem Cells, 2008, 26: 2634-2643

29 Trentin D, Hall H, Wechsler S, et al. Peptide-matrix-mediated gene transfer of an oxygen-insensitive hypoxia-inducible factor- $1 \alpha$ variant for local induction of angiogenesis. Proc Natl Acad Sci USA, 2006, 103: 2506-2511

30 Vincent K A, Shyu K G, Luo Y, et al. Angiogenesis is induced in a rabbit model of hindlimb ischemia by naked DNA encoding an HIF-1 $\alpha /$ VP16 hybrid transcription factor. Circulation, 2000, 102: 2255-2261

31 Baranova O, Miranda L F, Pichiule P, et al. Neuron-specific inactivation of the hypoxia inducible factor $1 \alpha$ increases brain injury in a mouse model of transient focal cerebral ischemia. J Neurosci, 2007, 27: 6320-6332

32 Sharp F R, Ran R, Lu A, et al. Hypoxic preconditioning protects against ischemic brain injury. NeuroRx, 2004, 1: 26-35

33 Gidday J M, Fitzgibbons J C, Shah A R, et al. Neuroprotection from ischemic brain injury by hypoxic preconditioning in the neonatal rat. Neurosci Lett, 1994, 168: 221-224

34 Ryan H E, Lo J, Johnson R S. HIF-1 $\alpha$ is required for solid tumor formation and embryonic vascularization. EMBO J, 1998, 17: 3005-3015

35 Tomita S, Ueno M, Sakamoto M, et al. Defective brain development in mice lacking the Hif-1 $\alpha$ gene in neural cells. Mol Cell Biol, 2003, 23: 6739-6749

36 Chang Y, Hsiao G, Chen S H, et al. Tetramethylpyrazine suppresses HIF- $1 \alpha$, TNF- $\alpha$, and activated caspase- 3 expression in middle cerebral artery occlusion-induced brain ischemia in rats. Acta Pharmacol Sin, 2007, 28: 327-333

37 Chen C, Hu Q, Yan J, et al. Multiple effects of 2ME2 and D609 on the cortical expression of HIF- $1 \alpha$ and apoptotic genes in a middle cerebral artery occlusion-induced focal ischemia rat model. J Neurochem, 2007, 102: 1831-1841

38 Helton R, Cui J, Scheel J R, et al. Brain-specific knock-out of hypoxiainducible factor- $1 \alpha$ reduces rather than increases hypoxic-ischemic damage. J Neurosci, 2005, 25: 4099-4107

39 Halterman M W, Federoff H J. HIF- $\alpha$ and p53 promote hypoxiainduced delayed neuronal death in models of CNS ischemia. Exp Neurol, 1999, 159: 65-72

40 Zhang $\mathrm{C} \mathrm{P}$, Zhu L L, Zhao T, et al. Characteristics of neural stem cells expanded in lowered oxygen and the potential role of hypoxiainducible factor- $1 \alpha$. Neurosignals, 2006, 15: 259-265

41 Zhao $\mathrm{T}$, Zhang $\mathrm{C} \mathrm{P}$, Liu Z H, et al. Hypoxia-driven proliferation of embryonic neural stem/progenitor cells-role of hypoxia-inducible transcription factor-1alpha. FEBS J, 2008, 275: 1824-1834

42 Xie L P, Qian X P, Gong S Y, et al. Analysis on the types of chemokines expressed by the murine thymic epithelial cell line MTEC1. Chin Sci Bull, 2000, 45: 1098-1101

43 Ma Q, Jones D, Borghesani P R, et al. Impaired B-lymphopoiesis, myelopoiesis, and derailed cerebellar neuron migration in CXCR4- and SDF-1-deficient mice. Proc Natl Acad Sci USA, 1998, 95: 9448-9453

44 Lazarini F, Tham T N, Casanova P, et al. Role of the alpha-chemokine stromal cell-derived factor (SDF-1) in the developing and mature central nervous system. Glia, 2003, 42: 139-148

45 Ceradini D J, Kulkarni A R, Callaghan M J, et al. Progenitor cell trafficking is regulated by hypoxic gradients through HIF-1 induction of SDF-1. Nat Med, 2004, 10: 858-864

46 Peng Z, Ren P, Kang Z, et al. Up-regulated HIF-1 [alpha] is involved in the hypoxic tolerance induced by 2 hyperbaric oxygen preconditioning. Brain Res, 2008, 1212: 71-78

Open Access This article is distributed under the terms of the Creative Commons Attribution License which permits any use, distribution, and reproduction in any medium, provided the original author(s) and source are credited. 